\title{
First Mathematics, Then Music: \\ J. S. Bach, Glenn Gould, and the Evolutionary Supergenius in The Outer Limits' “The Sixth Finger” (1963)
}

\author{
Reba A. Wissner
}

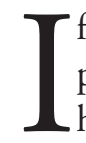

f asked to name a Western musical genius, a layperson would likely pick J. S. Bach. ${ }^{1}$ So common is the link between Bach and genius that his music is often used in film and television to represent a character's intelligence, both musical and intellectual. ${ }^{2}$ This is especially notable in the 1963 episode of The Outer Limits called "The Sixth Finger." In this episode, Gwyllm Griffiths (David McCallum) volunteers for a scientist who has found a way to advance man's evolution by over one million years, thereby creating human supergeniuses with an aptitude for rapid learning and enhanced mental capacity. For the purpose of this episode, a supergenius is one whose intelligence has evolved so far into the future that they are often misunderstood; they are considered abnormalities of nature. As a supergenius, Gwyllm has suddenly mastered mathematics

I am grateful to Christina Fuhrmann, Rebecca Fülöp, Naomi Graber, Eric Hung, Arianne Johnson Quinn, Linda Shaver-Gleason, and the anonymous reviewer for their insightful comments.

${ }^{1}$ The term "musical genius" is often problematic. One reason for this concerns the conflation of genius with creativity. See Edward E. Lowinsky, "Musical GeniusEvolution and Origins of a Concept, I," Musical Quarterly 50 (1964): 322. Another issue concerns a composer's capabilities in comparison with his contemporaries; if he surpasses his contemporaries, then he is often labeled a genius. For a discussion of this in the context of Josquin des Prez, see Paula Higgins, "The Apotheosis of Josquin des Prez and Other Mythologies of Musical Genius," Journal of the American Musicological Society 57 (2005): 444. As Christoph Wolff discusses, Bach himself, fluent in the mideighteenth-century discourse surrounding the newly discovered concept of genius, was a proponent of his own musical talent. This view was supported by his obituary, which stated that he was talented by nature, though its authorship is in question. See Christoph Wolff, "Defining Genius: Early Reflections of J. S. Bach's Self-Image," Proceedings of the American Philosophical Society 145 (2001): 475-76. For more on the problematic characterization of Bach as musical genius, see Hans Lenneberg, "The Myth of the Unappreciated (Musical) Genius,” Musical Quarterly 66 (1980): 230.

${ }^{2}$ Several notable examples of genius on television in the 1950s and 1960s include Luther Dingle (Burgess Meredith) in The Twilight Zone's "Mr. Dingle, the Strong" (1961), the Doctor (multiple actors) in Doctor Who (1963-1989), the Professor (Russell Johnson) in Gilligan's Island (1964-1992), and Mr. Spock (Leonard Nimoy) in Star Trek (1966-1969). 


\section{$64 \mathrm{BACH}$}

through his quick evolution; series co-creator Joseph Stefano felt that the next logical step would be the mastery of music. To represent this sudden musical aptitude, Stefano instructs in the script that Gwyllm should suddenly be able to play several preludes and a fugue from the WellTempered Clavier Book I. McCallum mimed his playing to Glenn Gould's then-new recording of the pieces, notable for Gould's rapid performance speed, to represent Gwyllm's sudden ability to play the piano. ${ }^{3}$

Many studies have explored Bach as a musical genius, the virtuosity necessary to play his music, and Gould's virtuosic, intellectual performances of Bach's music, but none have examined all three in tandem, especially in the context of a television episode. ${ }^{4}$ This essay discusses Bach's music as an embodiment of virtuosity and as a cultural representation of supergenius in "The Sixth Finger." I explore how Bach's music is used in a science fiction context to demonstrate these principles and how the choice of Gould's recording helps amplify them. I first examine what it means to be a genius and a supergenius, discussing the purported genius of both Gould and Bach. I then consider the use of Bach's music, and Gould's performance of it, to represent a supergenius. I seek to understand why, of all of the composers of Western art music, it is Bach's music that is used here to represent the intelligence and capabilities of a futuristic supergenius. The choice to use Bach's music in the visual context of television adds another dimension to the concept of genius, exemplifying both virtuosity and a specific composer whose music transcends time.

${ }^{3}$ J. S. Bach, The Well-Tempered Clavier, Book I, Preludes and Fugues 1-8, with Glenn Gould (piano), Columbia Masterworks ML 5808, 1963, 331/3 rpm. The remaining preludes and fugues were recorded in 1964-1965.

${ }^{4}$ For example, these sources discuss Bach as a musical genius: Joel Lester, "Heightening Levels of Activity and J. S. Bach's Parallel-Section Constructions," Journal of the American Musicological Society 54 (2001): 49-96; and Wolff, "Defining Genius," 474-81. These sources discuss Bach's virtuosity: David Schulenberg, The Keyboard Music of J. S. Bach (New York and Abingdon: Routledge, 2006); and Peter Williams, J. S. Bach: A Life in Music (Cambridge and New York: Cambridge University Press, 2007). These sources discuss Bach as both genius and virtuoso: Friedrich Blume and Wilburn W. Newcomb, "J.S. Bach's Youth," Musical Quarterly 54 (1968): 1-30; and Christoph Wolff, Johann Sebastian Bach: The Learned Musician (New York and London: W. W. Norton, 2000). These sources discuss Gould and his virtuosity: Georges Leroux, Partita for Glenn Gould: An Inquiry into the Nature of Genius, trans. Donald Winkler (Montreal: McGillQueen's University Press, 2010); and Peter F. Ostwald, Glenn Gould: The Ecstasy and Tragedy of Genius (New York and London: W. W. Norton, 1998). 


\section{The Definitions of Genius}

The idea of Bach as genius is prevalent. A study conducted in 1933 asked musicians from four major American orchestras to rank composers from the Western classical tradition by levels of genius. Although Beethoven won, Bach came in fifth place in a tabulation of all four of the orchestras combined. ${ }^{5}$ Similarly, in 1969, Paul R. Farnsworth asked musicologists to rank a list of 100 composers in order of who they believed was both the greatest genius and the most eminent composer. Bach ranked first. ${ }^{6}$ Both these studies demonstrate that Bach fulfills one of the most important definitions of genius: someone whose work has stood the test of time. ${ }^{7}$

But why has Bach acquired this reputation? What is genius? Much ink has been spilled about this topic, and perhaps Mark Kingwell defines it best: "The special quality of the genius is that he or she plots both the new trajectory and shows why the previous one was insufficient." 8 This definition explains why geniuses tend to be venerated for generations. It also explains why Bach was considered a genius: he often pushed the limits of existing genres further than what people thought possible. ${ }^{9}$ Another facet of genius is skill. This goes beyond talent, although both are fueled by creativity. ${ }^{10} \mathrm{E}$. J. Eysenk reminds us that the etymology of the word genius derives from the Latin word ingenium, "meaning both natural disposition and innate ability" (italics in original). ${ }^{11}$ In terms of music in particular, Kingwell writes that there is only a loose distinction between genius and talent. ${ }^{12}$ Estimates hold that technical competence in music takes 10,000 hours to achieve while excellence takes at least 30,000 hours. ${ }^{13}$ This excellence, when defined as genius, takes on almost superhuman qualities. Eysenk writes that a "'genius' is depicted as the possessor of a mystical gift that cannot be explained by the ordinary laws of human nature." 14 Geniuses are those who have attained,

${ }^{5}$ Emil E. E. Folgmann, "An Experimental Study of Composer-Preference of Four Outstanding Symphony Orchestras," Journal of Experimental Psychology 16 (1933): 716.

${ }^{6}$ The Social Psychology of Music, 2nd ed. (Ames: Iowa State University Press, 1969), 228.

${ }^{7}$ E. J. Eysenk, Genius: The Natural History of Creativity (Cambridge and New York: Cambridge University Press, 1995), 31.

${ }^{8}$ Glenn Gould (Toronto: Penguin Canada, 2009), 58. Emphasis in original.

${ }^{9}$ Williams, J. S. Bach, 80.

${ }^{10}$ Eysenk, Genius, 11-12.

${ }^{11}$ Genius, 13.

${ }^{12}$ Glenn Gould, 56.

${ }^{13}$ Paul Robertson, "What Is Musical Genius?," Clinical Medicine 8 (2008): 180.

${ }^{14}$ Genius, 11. 


\section{$66 \mathrm{BACH}$}

in the words of Paul Robertson, "technical mastery, virtuosity, emotional depth, integrity, originality, understanding, and transcendence" and, for some, also "exceptional memory and precociousness." 15

Specifically, for musical genius, these ideas highlight that Bach was not only a compositional genius but also a virtuoso, or "performer genius," and further, that it can require genius to perform Bach's music. Discussions of the distinction between talent and virtuosity come into play here. Francisco Montiero writes that "Some people see J. S. Bach also as a virtuoso, because of his virtues as a composer and as a performer: he not only composed pieces which reveal an incredible counterpoint technique but he also composed, played and improvised publicly virtuoso pieces (toccate, fantasie, suites)." ${ }^{\prime 6}$ It is here that we can refine what it means for Bach to be a genius: impeccable technique in both composing and performing pieces that were technically difficult, including the art of improvisation. We do know that Bach was hailed as a keyboard virtuoso throughout his life, a reputation that continued until well after his death and that is still present. ${ }^{17}$

To be considered a genius in general, however, rather than simply a gifted musician, the element of intellect is crucial. Several studies have grappled with finding a corollary between mental aptitude and musical ability with mixed results, often conflating musical genius with talent. As early as 1919, studies were published on the psychology of musical talent. Carl Emil Seashore's tome on the subject identified the correlation between intelligence and musical talent in elementary school children and found that skills such as pitch recognition could not adequately determine a child's intelligence level or academic ability. ${ }^{18}$ Other similar studies, such as that by George Cutler Fracker and Virgie M. Howard in 1928, performed on university students, supported the results of Seashore's study. ${ }^{19}$ In the same year as Fracker and Howard's study, Max Schoen outlined the intelligence attributes of those with musical talent:

15 "What Is Musical Genius?," 178.

16 "Virtuosity: Some (Quasi Phenomenological) Thoughts," in Proceedings of the International Symposium on Performance Science, ed. Aaron Williamon and Daniela Coimbra (Utrecht: Association Européene des Conservatoire, Académies de Musique et Musikhochschulen, 2007): 316.

${ }^{17}$ Wolff, Johann Sebastian Bach, 305-6.

${ }^{18}$ The Psychology of Musical Talent (Boston and New York: Silver, Burdett, and Co., 1919), 56.

19 "Correlation Between Intelligence and Musical Talent Among University Students," Psychological Monographs 39, no. 2 (1928): 160. 
We have evidence that in intelligence the musically talented person ranks above average. Specifically, he has a quick comprehension, a retentive memory, wide interests, power of concentration, ambition, alertness, originality and conscientiousness. The implication is that the possession of musical capacities above the average carries with it a high degree of general intelligence. ${ }^{20}$

All of these attributes tend to be associated not only with people who are considered musical geniuses but also with those who are geniuses in general. It is this high intelligence level that tends to set the bar for who is and is not considered a genius. Here again, Bach fits the definition of genius. Catherine Cox has estimated that Bach himself had an IQ of between 125 and 140; an IQ of over 120 is considered to indicate superior intellect. ${ }^{21}$

Connections between music and mathematics-an aptitude for which is often immediately linked to extreme intelligence-further link genius to the intellectual. Correlations between music and math go at least as far back as Martianus Cappella's early fifth-century treatise, The Marriage of Mercury and Philology. Cappella divides the seven liberal arts into two groups, one of which is the quadrivium of mathematical disciplines that includes music. Bach would have been aware of such associations between music and math and, indeed, may have included them in his music. Math, as David Rumsey notes, was something that was part of German musical life and therefore something with which Bach would have been well acquainted and would have encountered on a daily basis. ${ }^{22}$ Several scholars and performers have suggested that Bach was intrigued by numbers, numerology, and symbolism. ${ }^{23}$ Glenn Gould, also

20 “Musical Talent and Its Measurement," Musical Quarterly 14 (1928): 265.

${ }^{21}$ Genetic Studies of Genius, vol. 2 (Stanford, CA: Stanford University Press, 1926), cited in Farnsworth, The Social Psychology of Music, 155.

22 "Bach and Numerology: 'Dry Mathematical Stuff?," Sidney Society of Literature and Aesthetics 7 (1997): 146. For more on patterns and mathematics in Bach's music, see Rahul Siddharthan, "Music, Mathematics, and Bach: Pattern's in Bach's Music," Resonance 4, no. 4 (1999), 61-70. For more about math and symbolism in the WellTempered Clavier, see Herbert Anton Kellner, "Was Bach a Mathematician?," English Harpsichord Magazine 2, no. 2 (1978), http://www.harpsichord.org.uk/wp-content /uploads/2015/04/bachmath.pdf.

${ }^{23}$ See, for example, Eric Chafe, Analyzing Bach's Cantatas (Oxford and New York: Oxford University Press, 1999); Marjorie Wornell Engels, Bach's Well-Tempered Clavier: An Exploration of the 48 Preludes and Fugues (Jefferson, NC: McFarland and Co., 2014); Ruth Tatlow, Bach and the Riddle of the Number Alphabet (Cambridge and New York: Cambridge University Press, 1991); and Ruth Tatlow, Bach's Numbers: Compositional 
fond of numerology, famously admired Bach for his musical structures and textures. ${ }^{24}$ The tuning of the Well-Tempered Clavier demonstrates Bach's engagement with acoustical mathematics. ${ }^{25}$ Some have also argued that the patterning and structure of Bach's preludes and fugues in The Well-Tempered Clavier are mathematical. ${ }^{26}$ Peter Weir emphasized this in Picnic at Hanging Rock (1975), which uses the Prelude in C Major from the Well-Tempered Clavier Book I, specifically, in association with machination, automaticity, and patterning. ${ }^{27}$

The mathematical nature of Bach's music also brings to the fore questions of automation. Wendy Carlos's album, Switched on Bach (1968), in which Carlos plays Bach's music on the Moog synthesizer, is an example of both automation and the mechanical use of patterns in Bach's preludes. One study revealed that there is a correlation between elevated intelligence and the overlearning that leads to automaticity; especially in musicians, this can facilitate faster playing. ${ }^{28}$

Bach thus fulfills numerous definitions of genius. Using his music alone may have sufficed to embody genius in the Outer Limits episode. But another layer of genius is added when Glenn Gould's 1963 recording is used. Gould has been understood as a genius in a variety of ways over the course of his career. This narrative seems to stem from his father, who reported that as a child he not only seemed to have perfect pitch but also "would hum rather than cry and would reach his arms upward to 'flex

Proportion and Significance (Cambridge and New York: Cambridge University Press, 2016).

${ }^{24}$ David Neumeyer, "Underscore: Four Studies of the C Major Prelude," in Meaning and Interpretation of Music in Cinema, ed. David Neumeyer (Bloomington: Indiana University Press, 2015), 241.

${ }^{25}$ Ross Duffin and others have argued that Bach did not use equal temperament. See Ross Duffin, How Equal Temperament Ruined Harmony (and Why You Should Care) (New York: W. W. Norton, 2007), 40-46. For more on the possibilities of Bach's tuning, see also Bradley Lehman, "Bach's Extraordinary Temperament: Our Rosetta Stone: 1," Early Music 33 (2005): 3-23; Bradley Lehman, "Bach's Extraordinary Temperament: Our Rosetta Stone: 2," Early Music 33 (2005): 211-31; Mark Lindley and Ibo Ortigies, "BachStyle Keyboard Tuning," Early Music 34 (2006): 613-23; and Luigi Swich, "Further Thoughts on Bach's 1722 Temperament," Early Music 39 (2011): 401-8.

${ }^{26}$ See, for example, Herbert Anton Kellner, "Was Bach a Mathematician?," English Harpsichord and Keyboard Instrument Review 2, no. 2 (1978): 32-36.

${ }^{27}$ Neumeyer, "Underscore," 241.

${ }^{28}$ Benjamin S. Bloom, "The Hands and Feet of Genius: Automaticity," Educational Leadership 43, no. 5 (1986): 70-72. 
his fingers almost as if playing a scale." ${ }^{29}$ This unusual behavior laid the groundwork for the portrayal of Gould as a musical genius and might be linked to his distracting idiosyncrasies later in life, such as humming the melody, gesticulating and grimacing while playing, and adopting an unusual playing posture. ${ }^{30}$

Gould is also a performer who is foremost in the tradition of linking Bach's virtuosity with the idea of genius. In writing of Gould, Georges Leroux notes that there are two signposts of genius, both of which were exhibited by the pianist: "the transcendence implicit in any representation" and "freedom from convention." ${ }^{31}$ Gould is known for his technical tour de force, rapid-fire playing, and ability to interpret the music of Bach and other classical masters. In fact, before Gould's debut on American television in 1968, Leonard Bernstein introduced the pianist to the audience by saying, "Gould and Bach have become a kind of legendary combination." ${ }^{2}$ As Edward Said writes, "Gould's life-long association with the great contrapuntal genius [Bach] establishes a unique and interestingly plastic aesthetic space essentially created by Gould himself as intellectual and as virtuoso." ${ }^{33}$ David Neumeyer remarks that Gould is portrayed as a genius with a legacy by using the Prelude in C Minor from the Well-Tempered Clavier Book I in the film Thirty-Two Short Films About Glenn Gould, equating him directly with a virtuoso of Bach's caliber. ${ }^{34}$ This calls into question exactly where we can demarcate intelligence from virtuosity_or whether we can at all. Said believes that Gould's portrayal of Bach was as "a composer whose thinking compositions provided an opportunity for the thinking, intellectual virtuoso to try to interpret and invent or revise and rethink in his own way." ${ }^{35}$ Gould and Bach are thus intimately connected both with the idea of genius and with each other, and are therefore especially good choices for "The Sixth Finger."

${ }^{29}$ Kingwell, Glenn Gould, 45.

${ }^{30}$ For more on Gould's unique playing posture, see Edward Jones-Imhotep, "Malleability and Machines: Glenn Gould and the Technological Self," Technology and Culture 57 (2016): 293. For more on Gould's recordings of Bach throughout his career, see the article in this volume by Kristi Brown-Montesano, "Terminal Bach: Technology, Media, and the Goldberg Variations in Postwar American Culture," BACH: Journal of the Riemenschneider Bach Institute 50 (2019): 81-117.

${ }^{31}$ Partita for Glenn Gould, 66.

32 CBS Ford Presents, 31 January 1960.

33 “Glenn Gould, The Virtuoso as Intellectual," Raritan: A Quarterly Review 20, no. 1 (2000): 2.

34 "Underscore," 251.

35 "Glenn Gould," 13. 


\section{"The Sixth Finger" and the Supergenius}

"The Sixth Finger" ties into not only ideas of genius surrounding Bach and Gould, but also a long history of geniuses on television. Concepts of genius in television characters are similar to many of the qualities of genius discussed above. Geniuses have been depicted on television as "capable of amazing mental feats that run the gamut from astounding memory recall to impressive reasoning and deductive skills to remarkable facility with technology." 36 For example, Luther Dingle (Burgess Meredith) in The Twilight Zone's "Mr. Dingle, the Strong" (1961) is made a genius by visiting aliens from outer space. Aside from giving him superhuman strength, they give him superior intellect, including mathematical and statistical skills. Another example is Mr. Spock (Leonard Nimoy) from Star Trek: The Original Series (1966-1969), who combines his Vulcan logic with futuristic intelligence that is far more advanced than that of those around him. David Sidore writes that "The television landscape is full of surprisingly competent and hyperarticulate characters. In order to stand out as truly different, geniuses must be off the scale extraordinary." ${ }^{37}$ One study on media portrayals of geniuses demonstrates that some of the characteristics used to portray genius on television include advanced knowledge of the arts, including music, and being extraordinarily analytical, with an exceptional memory. ${ }^{38}$ There is an Othering, therefore, that occurs with television geniuses. ${ }^{39}$

"The Sixth Finger" takes these ideas of genius even further, into the realm of the supergenius. "The Sixth Finger" was the fifth episode of The Outer Limits to air, on 14 October 1963. The episode concerns a reclusive geneticist named Professor Mathers (Edward Mulhare) who is currently living in a remote part of Wales. He has become reclusive because he was partially responsible for the creation of the atomic bomb and severely regrets his role in the invention of such a damaging weapon. He has his groceries delivered to him by a teenager named Cathy Evans (Jill Haworth), whom he tells that he is working on a project that can

${ }^{36}$ Ashley Lynn Carlson, "Introduction," in Genius on Television: Essays on Small Screen Depictions of Big Minds, ed. Ashley Lynn Carlson (Jefferson, NC: McFarland and Co., 2015), 1.

37 "Spectacularly Ignorant: The Conflicted Representation of Genius," in Genius on Television, 16.

${ }^{38}$ Julie B. Wiest, "Entertaining Genius: U.S. Media Representations of Exceptional Intelligence," Media Tropes 6, no. 2 (2006): 158.

${ }^{39}$ Sidore, "Spectacularly Ignorant," 26. 
accelerate human evolution and create supergeniuses. He is currently using a monkey in his experiments and has already advanced its intellect by several hundred years. Cathy asks Mathers if he is willing to try his experiment on humans, which he affirms, and she volunteers. After running a test on her and finding out that she does not have the right blood type for the experiment, she sends her friend Gwyllm, a coal miner, to the scientist, and he too volunteers for the experiment. As Gwyllm has the proper blood type, Mathers then runs the experiment on him, making his intellect rapidly evolve into a superhuman 20,000 years into the future (the ultimate goal is to advance him intellectually one million years into the future). Because Gwyllm has evolved into a human 20,000 years into the future, the episode's writers make the case that those humans would be much more intellectually gifted than those of the present (here, 1963). This evolution gives him enhanced mental capabilities in all subjects, as well as telekinesis. He soon develops an enlarged brain and a sixth finger on each hand.

After the episode was filmed, the production team realized that the script for "The Sixth Finger" was ten minutes too short for the required 51-minute run time. Director James Goldstone suggested that to fill the remaining time, series co-creator Joseph Stefano construct a peripheral scene in which Gwyllm suddenly acquires a high level of skill in something else. Stefano felt that the best parallel to mathematics was music; thus, Gwyllm should suddenly learn how to play the piano at a level that illustrates extreme musical prowess, commensurate with his newfound supergenius. Prior to the scene in which Gwyllm plays several pieces to illustrate this new capability, Gwyllm goes through all of the books in Mathers's study and happens upon the score for the WellTempered Clavier.

Stefano wrote a five-page insert for the script. That same day, the production team decided to use Glenn Gould's then-new-and already quite popular-recording of Bach's Well-Tempered Clavier since Gould performed it faster than any other recording to date. The studio sent a prop person down to the local music store, Wallich's Music City, for a copy of the record. McCallum then learned to mime the playing of three preludes and one fugue, partially to avoid having to hire a stand-in pianist and partially because Goldstone conceived of the camera angles 
such that the camera would pan upward from McCallum's hands to his face in a single shot. ${ }^{40}$

In the final version, three different preludes were used, all from Book I: Prelude in C Minor, Prelude in D Major, and Prelude in C Major. ${ }^{41}$ $\mathrm{He}$ also plays the Fugue in D Major. According to McCallum, the four pieces were chosen based on how quickly he thought he could learn to mime the recording. For McCallum, learning to mime the playing was relatively easy. He was a trained oboist who had studied at the Royal Academy of Music, and his father was a concert pianist and conductor, so he was likely well acquainted with both the keyboard and Bach's WellTempered Clavier and could easily mime the rhythms on the piano. He took the record home with him to practice miming the fingering as well as he could and then returned to the set later that day to film the scene. ${ }^{42}$ As McCallum explained,

I remember feeling a little responsible for suggesting that sequence. We came up with the idea of having Gwyllm play the piano in the night, having learned very fast. One scene had me looking through book after book; it was shot over my shoulder as I was turning pages, quite fast. And I found an edition of Bach preludes, flipped open to the music, and paused just a split second. ${ }^{43}$

The score as object comes to the fore in the episode. We see the score in the shot in several ways. First it appears through an extreme close-up (fig. 1), and then as Gwyllm plays it in his study when Mathers comes in to see what he is doing (fig. 2). Seeing the score in the shot makes it easier for us to understand the technique necessary for Gwyllm to play the piece, given its rapid running sixteenth notes. Even the extreme closeup of the score shows its level of technical difficulty; the viewer may

40 David J. Schow, The Outer Limits Companion, 2nd ed. (Hollywood: GNP Crescendo, 1998), 98.

${ }^{41}$ For an in-depth theoretical examination of Gould's recording of the Preludes in C Minor and C Major, see Daniel Barolsky and Peter Martens, "Rendering the Prosaic Persuasive: Gould and the Performance of Bach's c-minor Prelude (WTC I)," Music Theory Online 18, no. 1 (2012), http://www.mtosmt.org/issues/mto.12.18.1 /mto.12.18.1.barolsky_martens.php; and Nicholas Cook, "Structure and Performance Timing in Bach's C Major Prelude (WTC I): An Empirical Study," Music Analysis 6 (1987): 257-72. It is important to note that this is not the only time this recording of Gould's version of the Prelude in C Minor from the Well-Tempered Clavier Book I was used on screen; it also appeared in Thirty-Two Short Films About Glenn Gould and the 2003 film The Triplets of Belleville.

${ }^{42}$ Schow, The Outer Limits Companion, 98.

${ }^{43}$ Schow, The Outer Limits Companion, 98. 
Figure 1: Close-up of the Prelude in D Major from the Well-Tempered Clavier Book I in "The Sixth Finger"

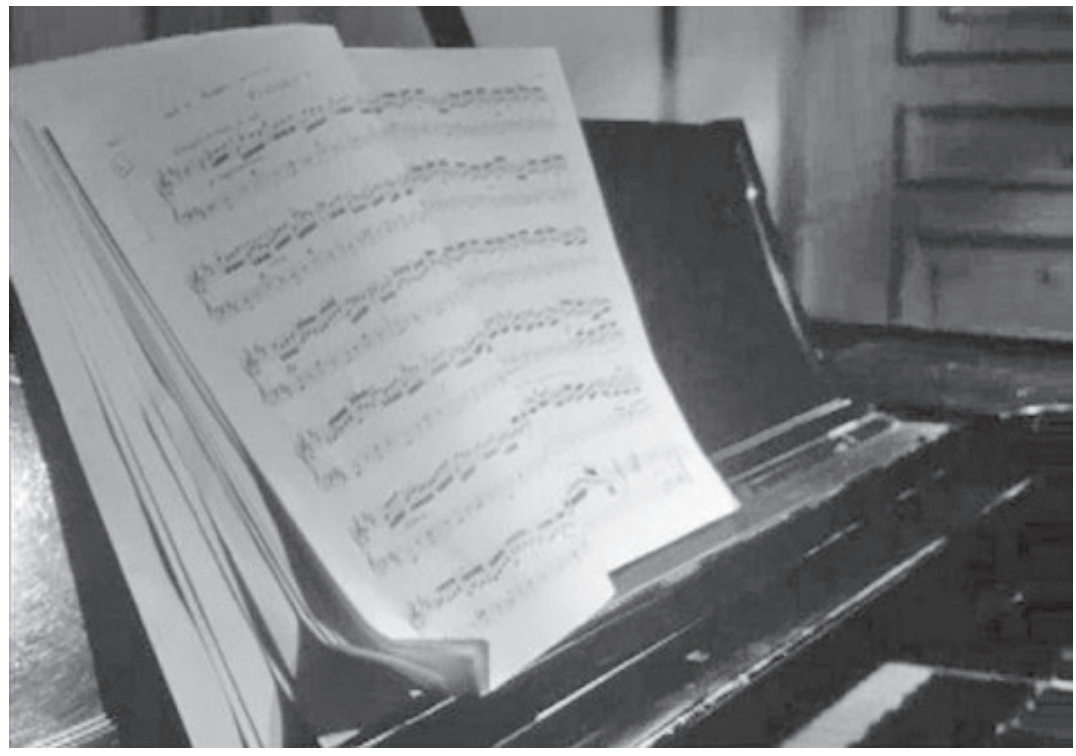

Figure 2: Gwyllm playing the Prelude in D Major from the Well-Tempered Clavier Book I in "The Sixth Finger"

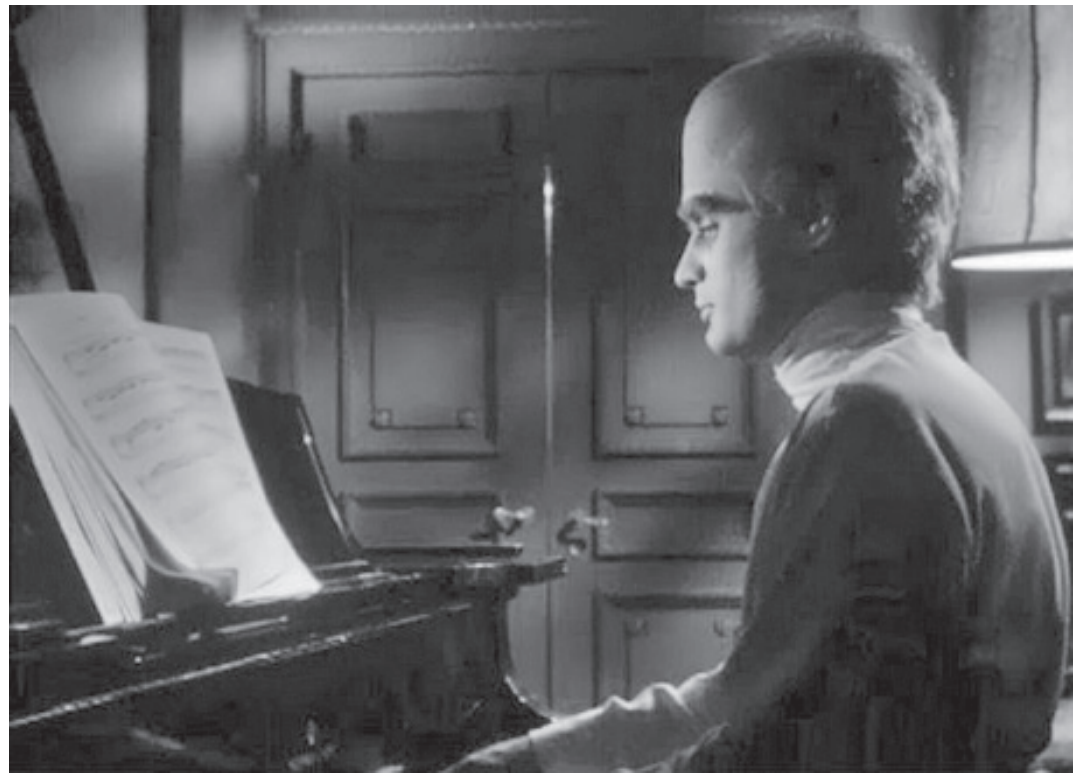


not be able to read music, but they can certainly see a large number of notes on the page and Gwyllm's fingers moving rapidly as he reads the score, which follows the common visual tropes of seeing the apparatus of genius. It emphasizes just how difficult this piece is to play even with practice, making his ability to play suddenly, seemingly by osmosis, all the more remarkable.

Visual representations of genius, especially on television, "tended to skew attention towards the artist's face and hands," making it unusual to focus on the performer's body. ${ }^{44}$ The visual focus usually concentrates on the apparatus of the person's genius, whether it be a set of test tubes, a blackboard with a mathematical formula, or a musical score. In this case, however, we do see Gwyllm's face, hands, and body, as well as the score; the latter is the physical representation of his newfound supergenius, allowing us to see the printed information that conveys what Gwyllm needs to perform on the piano. It is likely that the focus on Gwyllm's body is to illustrate his unusual form given his enlarged cranium.

This episode emphasizes the idea of genius - and supergenius-in several ways and can also help us define the concept of genius. One can question how an examination of Bach's music, paired with the extreme intellect represented in a science fiction television episode, helps us arrive at a concrete definition of supergenius. In the context of "The Sixth Finger," the quick process of intelligence evolution beyond that of a normal person is one contributing factor. The process of gaining knowledge, in general, tends to be a slow one, but here, it is almost instantaneous. Another contributing factor is the intelligence level itself. To my knowledge, there have not been any studies that suggest that intelligence levels increase from one century to the next, but the premise of this episode is that this does occur, and that humans from that far in the future therefore would not simply be geniuses, but would have evolved into supergeniuses.

The episode also plays into contemporary beliefs about intelligence and brain size, which we now know to be false. Today, studies have linked certain brain markers to musical ability but have also shown that there are no structural brain differences in adults or children between musicians and non-musicians and have found nothing that indicates that brain size is proportionate to intelligence. ${ }^{45}$ In the 1950 s-1970s, however, several

${ }^{44}$ Graham Carr, "Visualizing 'The Sound of Genius': Glenn Gould and the Culture of Celebrity in the 1950s," Journal of Canadian Studies 40, no. 3 (2006): 24.

${ }^{45}$ Andrea Norton, Ellen Winner, Karl Cronin, Katie Overy, Dennis J. Lee, and 
studies did link brain size to intelligence and suggested that as humanity becomes more cultured, humans become more intelligent. ${ }^{46}$ The episode plays directly into these ideas. Gwyllm's skull has enlarged upon his evolution because the size of his brain is ostensibly directly proportional to the level of intelligence; this can already be seen in the profile shot in figure 2. ${ }^{47}$ Thus, this is a physical manifestation of Gwyllm's supergenius that is visible as he suddenly is able to play the piano. His whole body here is also shown in order to emphasize the proportion of his head size to his body.

As Gwyllm becomes more intelligent throughout the episode, not only do his brain and skull grow, but so too does a sixth finger develop on each hand. It is implied that the addition of this sixth finger allows Gwyllm to play the piano more easily than he would have been able to with only five fingers. Thus, the addition of a new manual dexterity skill is tied directly to his brain size, allowing him to take on new physical and mental tasks with virtual ease, all thanks to his rapid evolution. But perhaps this is another reason they chose not to do a close-up of his hands; it might have been too technologically difficult to show the sixth finger actually working.

\section{Why Bach?}

"The Sixth Finger" could have used a wide variety of music to demonstrate Gwyllm's supergenius. So why Bach? Why would Gwyllm gravitate toward the score of the Well-Tempered Clavier and not toward the works of another composer, assuming there are other piano scores in the music room? As argued earlier, Bach embodies several facets of genius, virtually all of which the episode draws on to underline Gwyllm's sudden transformation.

The first is the idea of timelessness and universality. Robert Marshall emphasizes Bach's transcendence: "Bach's breathtaking musical synthesis,

Gottfried Schlaug, "Are There Pre-Existing Neural, Cognitive, or Motoric Markers for Musical Ability?," Brain and Cognition 59 (2005): 129.

${ }^{46}$ Sheldon C. Reed, "The Evolution of Human Intelligence: Some Reasons Why It Should Be a Continuing Process," American Scientist 53 (1965): 320.

${ }^{47}$ For more on this, see, for example, D. O. Hebb, "Intelligence, Brain Function, and the Theory of Mind," Brain 82, no. 2 (1959): 260-75; Reed, "The Evolution of Human Intelligence," 317-26; Leigh van Valen, "Brain Size and Intelligence," American Journal of Physical Anthropology 40, no. 3 (1974): 417-23; and Lee Willerman, Robert Schultz, J. Neal Rutledge, and Erin D. Bigler, "In Vivo Brain Size and Intelligence," Intelligence 15, no. 2 (1991): 223-28. 
unifying as it does a vast array of historical and national styles, can be seen as an effort to transcend the cultural limitations of geography and history, of time and place, in order to create a 'universal' artwork." ${ }^{48}$ A discussion of the exchange between Gwyllm and the Professor is pertinent here. When the Professor enters the room, Gwyllm thanks him for giving him the ability to observe his culture-directed at Mathers with the "your" preposition-from a man 20,000 years into the future as he plays the Prelude in C Minor. The use of "your" culture here is interesting, showing that Gwyllm does not consider himself a part of the same culture as Mathers; the implication is that those less intelligent than the supergenius Gwyllm are culturally different. Still, even in Gwyllm's advanced state, Bach is considered a genius. Gwyllm says to Mathers, "Amazing, isn't it? The things that endure the ravages of time and taste? This simple prelude, for instance. Bach will quite probably outlive us all." Gwyllm also connects the idea of creation, of forging something both new and enduring, with timelessness. He says, "Man produces little that is lasting. Truly lasting. It's understandable. Fear, conformity, immorality, these are heavy burdens, great drainers of creative energy and when we are drained of creative energy we do not create. We procreate, but we do not create," during which he plays the Prelude in D Major followed by the Fugue in D Major. Shortly before the episode aired, some scientists argued that intelligence was a genetic mutation that stemmed from human evolution, and that that evolution was based on cultural advancement. ${ }^{49}$ There is a suggestion that Bach's music not only transcends cultural and temporal limitations, but may even have planted the seed that would allow humans eventually to evolve to Gwyllm's supergenius state.

The connection among mathematics, music, and genius surfaces in a surprising context: a discussion of Gwyllm's sudden physical skill in piano playing. Professor Mathers asks him if he has always been able to play the piano this well, to which Gwyllm replies that he has "never before touched the keys of a piano." The added sixth finger has of course helped him acquire greater physical abilities, but Gwyllm asserts that the ability to play the piano is nothing more than understanding mathematics and having a certain degree of manual dexterity. Here, the trope of combining mathematical genius and musical genius is elided with the trope of Bach as a musically mathematical genius.

48 "Bach and Mozart: Styles of Musical Genius," BACH: The Journal of the Riemenschneider Bach Institute 22, no. 1 (1991): 31.

${ }^{49}$ Reed, “The Evolution of Human Intelligence," 320. 
Clearly, there are many reasons why Bach was an appropriate choice for a supergenius. Perhaps it was because the Well-Tempered Clavier was often considered groundbreaking. ${ }^{50}$ As mentioned earlier, the ability to tune a keyboard instrument to play well in all keys was unusual. It is also fitting to consider the title page, on which Bach wrote "for the Use and Profit of the Musical Youth Desirous of Learning." Indeed, the composer always maintained the conviction that music should be used to educate. ${ }^{51}$ The entire crux of "The Sixth Finger" is based on intelligence and a desire to learn in the guise of the rapid evolution of intelligence. The reason that Gwyllm initially goes to the piano is because he has already read every single book in his study in a matter of hours and has already mastered mathematics.

Why these particular portions of the Well-Tempered Clavier? The answer might be as simple as their placement as the first three preludes in the book. These pieces are also frequently assigned to intermediate or early advanced piano students. We must consider that the typical audience for an Outer Limits episode comprised mainly teenagers, some of whom might have been playing one of those pieces for their piano lessons at the time of the episode premiere. Joel Lester also notes that these three preludes, in addition to the Prelude in E Minor, "form a 'mini-series' within Volume 1 of the Well-Tempered: all contain recurring figurations over very similar basses." ${ }^{52}$ The rhythms in each of the three preludes are similar and once again illustrate Bach's penchant for patterns. For the Prelude in C Minor, these groupings are reflected in the left hand with the notes moving in contrary motion, but the voices in the Prelude in $\mathrm{C}$ Major move in oblique motion. David Schulenberg points out that the Prelude in C Major "can be played energetically, enough to make the little flourish at the end sound brilliant." 33 Thus, watching Gwyllm's hands at the speed at which he mimes the pieces gives the illusion to the untrained musician that they are difficult to play. The issue of miming may also be why the preludes were chosen and only one of their paired fugues. Since fugues are equated with musical and technical mastery, more so than the preludes, it is odd that only one is used. This is probably a technical issue; it is likely that miming the fingering for more fugues was beyond McCallum's capabilities.

${ }^{50}$ Wolff, “Defining Genius,” 480.

${ }^{51}$ Marshall, "Bach and Mozart," 19.

52 "J. S. Bach Teaches Us How to Compose: Four Pattern Preludes of the WellTempered Clavier," College Music Symposium 38 (1998): 33.

${ }^{53}$ The Keyboard Music of J. S. Bach, 207. 
It is also important to ask why Gould's performance of these pieces was chosen. As described earlier, Gould's connections to both genius and Bach likely played a role. But his specific performance choices probably also influenced the choice. Two of the three preludes - C Major and C Minor-are played much quicker than usual, giving the impression that the music is much more difficult than it really is, while the third, the Prelude in D Major, is played slower than usual. These three preludes are teaching pieces that really are not that virtuosic (compared with Bach's organ fugues or the Goldberg Variations). Gould himself rather dismissively describes the Preludes in C Major and C Minor as "prosaically prefatory ... perhaps falling within that etudish category." ${ }^{54}$ Yet, the fact that the pieces are more easily recognizable to the audience, combined with the fact that they are played faster than expected, makes them more effective at portraying supergenius than a piece that is inherently virtuosic but unfamiliar. In this case, Gould's faster tempo is useful for depicting extreme genius because the average viewer cannot be expected to differentiate between a mediocre and a "great" performance. Speed, therefore, is a handy shorthand here for talent and genius. Fast playing tends to be perceived as virtuosic, even if it is not necessarily virtuosic, because it hides imperfections and gives the illusion of a flawless performance. ${ }^{55}$ The scene was written around the music, so there was no practical reason why this recording was chosen.

Yet, there is also an element of introspection. The Prelude in D Major is played much slower than the other three pieces, giving Gwyllm a sense of contemplation not found often in this scene. Similarly, in playing the Prelude in D Major, Gwyllm states that he has evolved beyond basic emotions, but Gould's playing represents the opposite of this. Of all of the pieces used in the episode, this one is played most introspectively, expressing the emotion that Gwyllm is supposedly no longer able to express and that perhaps Gould himself was trying to downplay. Some of the other preludes, however, have characteristics that make them seem more difficult than they are. As Daniel Barolsky and Peter Martens note, for example, the Prelude in C Minor is filled with hypermeter; that is, each measure functions as a single beat rather than

54 "The Art of the Fugue," in The Glenn Gould Reader, ed. Tim Page (New York: Alfred A. Knopf, 1989), 21.

${ }^{55}$ Antoine Hennion, “'As Fast as One Possibly Can ...': Virtuosity, a Truth of Musical Performance," in Critical Musicological Reflections. Essays in Honour of Derek B. Scott, ed. Stan Hawkins (Aldershot: Ashgate, 2012), 128. 
each individual note serving to contribute to a beat within the measure. ${ }^{56}$ Gould's performance of the prelude, with his rapid speed and pedaling technique, however, blurs the hypermeter. This lack of precision would be easier for McCallum to mime, but it also adds a level of complexity to the voicing that may have been interpreted as technologically masterful.

Glenn Gould's recording of the Well-Tempered Clavier was also recent at the time, and it is likely that most people would not have heard it yet (despite its popularity), making the pianist's interpretation brand-new to many viewers. It was unlike other recordings of the work by other pianists and therefore more intriguing and complex, making it easy for the viewer to suspend disbelief and believe that the playing was part of Gwyllm's new skill. To be sure, the viewers would not have known it was mimed from a recording, and some may have interpreted it as being played live by McCallum; his miming was highly believable, right down to depressing the proper keys at the right time with the proper fingering. ${ }^{57}$

Even if listeners did not yet know this recording, they knew Gould. Some people even considered his skill otherworldly, which makes it especially appropriate for Gwyllm's playing of Bach. Gwyllm's sudden skill makes him an outsider, and the combination of Bach's music and Gould's interpretation of it ties the portrayal of Gwyllm's playing of Bach together. In the case of this episode, the "otherworldliness" of Gould's playing is joined with Gwyllm's "outer limits"-ness; like Gould, he is something that can be considered a force of nature - or at the very least an anomaly of nature.

\section{Conclusion}

The appearance of Bach's music is not unusual in film and television-his pieces have appeared in, to name only a few examples, the films of Andrei Tarkovsky and Pier Paolo Pasolini, and on television in The Simpsons and The Twilight Zone-but its connection with and use in science fiction is a little more unusual. In the case of "The Sixth Finger," Bach's music represents not only supreme intelligence and musical prowess, but also high culture.

By examining Bach and Gould as musical geniuses, and the virtuosic, intellectual performances of Bach's music in the context of a television episode about genius, we can better understand how genius

\footnotetext{
56 "Rendering the Prosaic Persuasive."

${ }^{57}$ I am grateful to Arianne Johnson Quinn for pointing this out to me.
} 
was conceived in 1960s television. Genius - especially extreme geniustended to be overexaggerated. The best way to do that visually was by portraying someone who was as physically evolved as intellectually; the best way to do that musically was by using a composer known to be technically difficult to illustrate that person's sudden musical prowess.

As "The Sixth Finger" has shown, when it comes to using Bach to represent a futuristic supergenius, the secret lies in the renowned technical complexity and mastery of his work, combined with the rapid playing speed. When one thinks of a musical genius, they typically think of someone who is a virtuoso player or composer. But no matter your interpretation of the use of Bach's music here to represent genius, Gwyllm does have at least one thing right: Bach will quite probably outlive us all.

\section{Abstract}

In a 1963 episode of The Outer Limits called "The Sixth Finger," Gwyllm Griffiths (David McCallum) volunteers for a scientist who has found a way to advance man's evolution by over one million years, thereby creating human supergeniuses with an aptitude for rapid learning and enhanced mental capacity. The final script was ten minutes too short for its time slot, so the production team had to come up with an inexpensive way to fill that space. Since Gwyllm has mastered mathematics, series co-creator Joseph Stefano felt that the next logical step would be mastery of music. Stefano immediately wrote a five-page insert in which Gwyllm plays several preludes and a fugue from the Well-Tempered Clavier Book I since he felt they represented musical genius. The production team chose Glenn Gould's then-new recording of the pieces, notable for Gould's rapid performance speed. Gwyllm then mimed playing at this accelerated pace to represent his sudden musical adeptness.

Studies have been conducted on Bach as a musical genius, the virtuosity necessary to play his music, and Gould's virtuosic, intellectual performances of Bach's music, but none have examined all three in tandem, especially in the context of a television episode. This essay discusses Bach's music as an embodiment of virtuosity and as a cultural representation of genius in "The Sixth Finger." The choice to use Bach's music in the visual context of television adds another dimension to the concept of genius, exemplifying both virtuosity and a specific composer whose music transcends time. 